\title{
Fast and direct measurements of the electrical properties of graphene using micro four-point probes
}

Klarskov, Mikkel Buster; Dam, Henrik Friis; Petersen, Dirch Hjorth; Hansen, Torben Michael; Löwenborg, A; Booth, Tim; Schmidt, Michael Stenbæk; Lin, R; Nielsen, P F; Bøggild, Peter

Published in:

Nanotechnology

Link to article, DOI:

$10.1088 / 0957-4484 / 22 / 44 / 445702$

Publication date:

2011

Document Version

Publisher's PDF, also known as Version of record

Link back to DTU Orbit

Citation (APA):

Klarskov, M. B., Dam, H. F., Petersen, D. H., Hansen, T. M., Löwenborg, A., Booth, T., Schmidt, M. S., Lin, R., Nielsen, P. F., \& Bøggild, P. (2011). Fast and direct measurements of the electrical properties of graphene using micro four-point probes. Nanotechnology, 22(44), 445702. https://doi.org/10.1088/0957-4484/22/44/445702

\section{General rights}

Copyright and moral rights for the publications made accessible in the public portal are retained by the authors and/or other copyright owners and it is a condition of accessing publications that users recognise and abide by the legal requirements associated with these rights.

- Users may download and print one copy of any publication from the public portal for the purpose of private study or research.

- You may not further distribute the material or use it for any profit-making activity or commercial gain

- You may freely distribute the URL identifying the publication in the public portal 
Fast and direct measurements of the electrical properties of graphene using micro four-point probes

This article has been downloaded from IOPscience. Please scroll down to see the full text article.

2011 Nanotechnology 22445702

(http://iopscience.iop.org/0957-4484/22/44/445702)

View the table of contents for this issue, or go to the journal homepage for more

Download details:

IP Address: 192.38.67.112

The article was downloaded on 07/11/2011 at 09:53

Please note that terms and conditions apply. 


\title{
Fast and direct measurements of the electrical properties of graphene using micro four-point probes
}

\author{
M B Klarskov ${ }^{1}$, H F Dam ${ }^{1}$, D H Petersen ${ }^{1}$, T M Hansen ${ }^{1}$, \\ A Löwenborg ${ }^{1}$, T J Booth ${ }^{1}$, M S Schmidt ${ }^{1}$, R Lin ${ }^{2}$, P F Nielsen ${ }^{2}$ and \\ P Bøggild ${ }^{1}$ \\ ${ }^{1}$ Department of Micro- and Nanotechnology, Technical University of Denmark, \\ DTU Nanotech, Building 345E, DK-2800 Kongens Lyngby, Denmark \\ ${ }^{2}$ CAPRES A/S, Scion-DTU, Building 373, DK-2800 Kongens Lyngby, Denmark \\ E-mail: mikkel.olesen@ nanotech.dtu.dk
}

Received 8 July 2011, in final form 14 September 2011

Published 6 October 2011

Online at stacks.iop.org/Nano/22/445702

\begin{abstract}
We present measurements of the electronic properties of graphene using a repositionable micro four-point probe system, which we show here to have unique advantages over measurements made on lithographically defined devices; namely speed, simplicity and lack of a need to pattern graphene. Measurements are performed in ambient, vacuum and controlled environmental conditions using an environmental scanning electron microscope (SEM). The results are comparable to previous results for microcleaved graphene on silicon dioxide $\left(\mathrm{SiO}_{2}\right)$. We observe a pronounced hysteresis of the charge neutrality point, dependent on the sweep rate of the gate voltage; and environmental measurements provide insight into the sensor application prospects of graphene. The method offers a fast, local and non-destructive technique for electronic measurements on graphene, which can be positioned freely on a graphene flake.

(Some figures in this article are in colour only in the electronic version)
\end{abstract}

\section{Introduction}

Since the first demonstration of isolated graphene and its field effect properties [1], much effort has been focused on its electrical properties [2], since the carriers in graphene behave as massless fermions with a linear dispersion relation around the Dirac points [3].

The typical approach toward testing the electrical properties of graphene is by patterning the graphene into a suitable geometry and depositing electrodes by photo- or electron beam (e-beam) lithography $[1,4,5]$. In the case of microcleaved graphene, the lithography pattern must be aligned to the individual graphene flake, followed by at least one round of exposure, development, metallization and lift-off. Large-area graphene from chemical vapor deposition (CVD) and epitaxial growth on silicon carbide is less restrictive in terms of alignment, but still requires definition of a field effect transistor-like electrode geometry [6].
With the drastic increase in available graphene area [7, 8], the verification of the electronic properties of as-produced graphene becomes increasingly important, and will constitute a quickly narrowing bottleneck for industrial development and research. Furthermore, the lithographic patterning of graphene leads to irreversible changes to the sample, and previous results show that the lithography process itself may change the electronic properties, leaving resist residues, contaminants etc $[9,10]$.

An obvious solution is to use repositionable electrodes. Several scanning probe techniques have been applied to the characterization of graphene, such as scanning probe gate measurement of the charge neutrality point [11], and electrostatic force microscopy [12]. Conducting atomic force microscopy (AFM) is also possible [13]; however, here it was demonstrated that the bias voltage can lead to undesirable patterning of the graphene through local anodic oxidation [13]. Although these methods probe the 


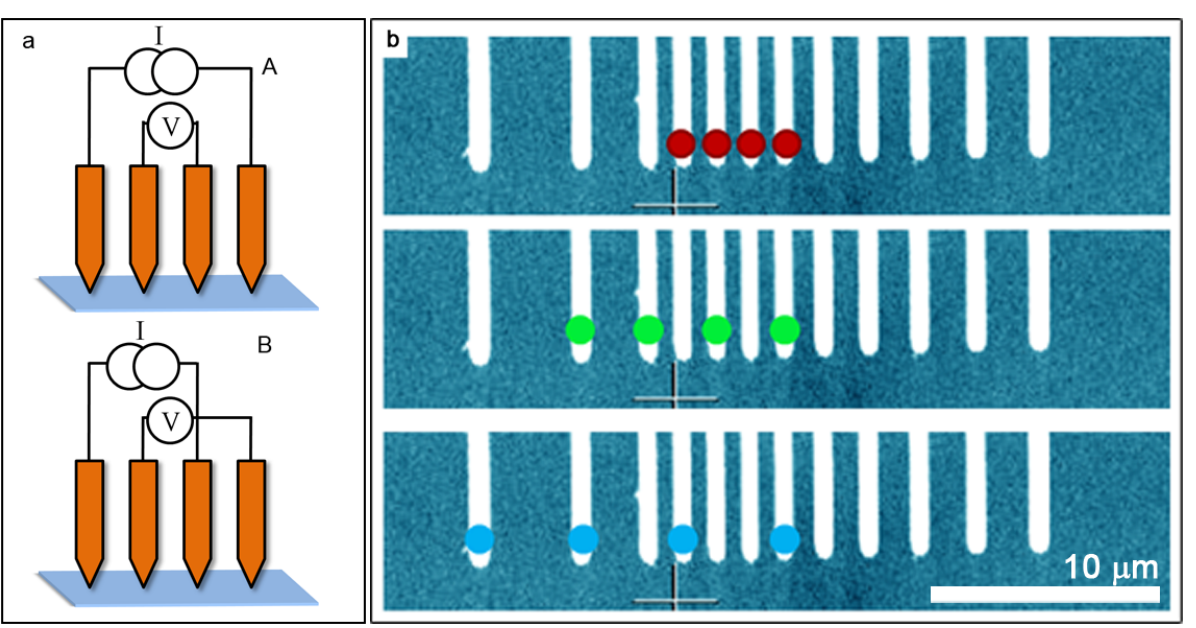

Figure 1. (a) Illustration of M4PP with two different measurement configurations, A and B, where $V$ measures the voltage drop, and $I$ indicates a current source. (b) Optical image of the 12-point probe used in ambient measurements. Three different pitches are indicated, $1.5 \mu \mathrm{m}, 3.0 \mu \mathrm{m}$ and $4.5 \mu \mathrm{m}$, respectively.

electrical properties, lithographic definition of at least one electrode on the graphene sample is still required. Recently, four independently positioned electrodes were applied for rotational square configuration measurements of multi-layer epitaxial graphene on $\mathrm{SiC}$ in a $100 \times 100 \mu \mathrm{m}^{2}$ measurement area [14]. Although this technique allows for anisotropic conductance characterization [15], microfabricated four-point probes (M4PP) [16] are much simpler to operate, and do not involve individual alignment of the scanning probe tips. This makes mapping a practical possibility, which has been demonstrated for numerous other materials including conjugated monolayer polymers [17] and non-uniform ultrathin semiconductors with lateral dimensions down to $10 \times$ $10 \mu \mathrm{m}^{2}$ [18]. In addition, it has recently been shown that the Hall carrier mobility and carrier density can be extracted from collinear four-point probe measurements [19]. Multiple previous measurements of the conductance of graphene using lithographically defined fixed electrodes have been made, falling in the range $0.1-8 \mathrm{mS}$ [20-22], and the conductivity of graphene particles has been measured at $64 \mathrm{mS} \mathrm{cm}^{-1}$ using standard four-point probe techniques [23].

In this work we show that repositionable, monolithic micro four-point probes provide a fast, local and nondestructive technique for measuring the electrical properties of graphene, despite the extreme thinness of the material and the mechanical contact required. We show that the method can be used in both ambient and vacuum conditions, and employ environmental scanning electron microscopy (ESEM) to investigate the effect of the local environment.

\section{Methods}

The current investigation involves equidistant M4PP measurements on micromechanically cleaved graphene samples. Two electrodes are used for sourcing a current, $I$, and the other two for measuring the corresponding voltage drop, $V$. Two different measurement modes were used; single and dual configuration. In the single configuration mode we calculate the sheet conductance $\sigma=I /(c V)$, where $c$ is the geometrical correction factor [24]. Due to position errors of the electrodes there is a unique static error on the geometrical correction factor. Using dual configuration, this static position error is eliminated [25], as well as geometrical errors caused by finite sample size [27]. Figure 1(a) illustrates the two current/voltage electrode configurations used for dual configuration position correction, A and B.

Graphene samples were produced by mechanical exfoliation of graphite on low-resistivity wafers with thermally grown $90 \mathrm{~nm}$ silicon dioxide $\left(\mathrm{SiO}_{2}\right)$ film. Prior to the exfoliation, the wafers were baked at $120^{\circ} \mathrm{C}$ and cleaned in $\mathrm{O}_{2} / \mathrm{N}_{2}$ plasma for 5 min. Gate voltage was controlled by a back-side contact; however, to improve M4PP to surface alignment, top-side connections were preferred. To avoid chemical interaction with the graphene, the top electrode was made by removing an area of the $\mathrm{SiO}_{2}$ by reactive ion etching and metal deposition using a shadow mask.

\section{Ambient conditions}

The measurements in ambient conditions (i.e. in air at room temperature and pressure) were obtained using a commercial Capres MicroRSP-M150 system with Capres M12PP probes (see figure 1(b)), from which the equidistant pitch configurations $1.5,3$ and $4.5 \mu \mathrm{m}$ were selected for the measurements. An AC current of $1 \mu \mathrm{A}$ was used. The time for making a measurement was $5 \mathrm{~min}$, with a gate voltage sweep from $0 \mathrm{~V}$ to $+60 \mathrm{~V}$ and back to $0 \mathrm{~V}$.

Figure 2 displays a typical dual configuration measurement of the conductance as a function of applied gate voltage in ambient conditions. Three different pitches were used, indicated by the three different curves, and two different measurement configurations were combined $[25,26]$ to give the result shown. Defining hysteresis as the difference in the conductance minima, we observe a pronounced hysteresis of approximately $35 \mathrm{~V}$. Such hysteresis effects are commonplace 


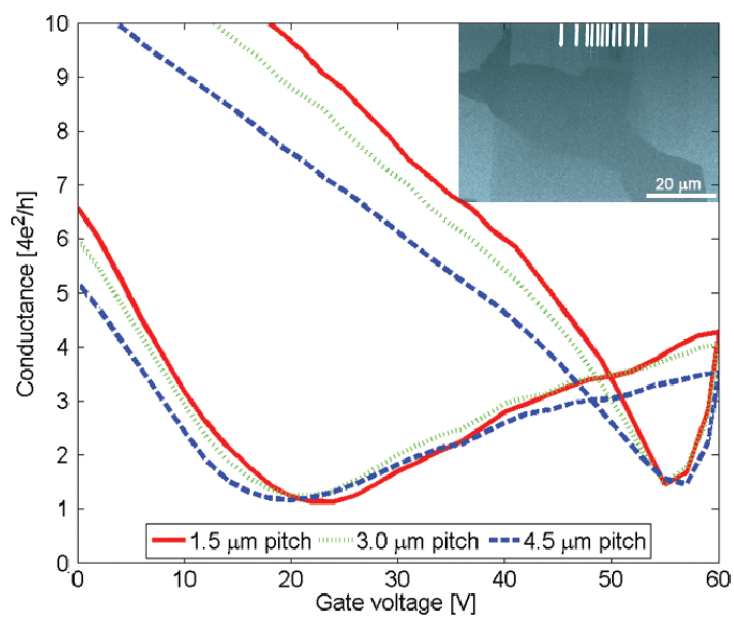

Figure 2. The conductance of a graphene flake in ambient conditions on clean $\mathrm{SiO}_{2}$ as function of applied gate voltage. The conductance is measured with 10 configurations per gate step and gate voltage steps of $2 \mathrm{~V}$. In each data point, recordings with three different voltage probe pitches, $1.5 \mu \mathrm{m}$ (red solid), $3.0 \mu \mathrm{m}$ (green dotted) and $4.5 \mu \mathrm{m}$ (blue dashed), were done. The insert shows an optical image of a 12-point probe over a single layer of graphene.

for graphene on $\mathrm{SiO}_{2}$ [4, 5, 27-29]. The effect was explained by Wang et al [30] as trapped charges in the $\mathrm{SiO}_{2}$ leading to a different effective gate voltage experienced by the graphene, as compared to the applied gate voltage. The hysteresis effect is further discussed after vacuum measurements. The carrier mobility was calculated from $\mu=t / \varepsilon \times \mathrm{d} \sigma / \mathrm{d} V_{\mathrm{g}}$ [1], where $t$ is the thickness and $\varepsilon$ is the permittivity of $\mathrm{SiO}_{2}$ and $V_{\mathrm{g}}$ is the gate voltage. The mobilities are calculated to be in the range $1000-2500 \mathrm{~cm}^{2} \mathrm{~V}^{-1} \mathrm{~s}^{-1}$.
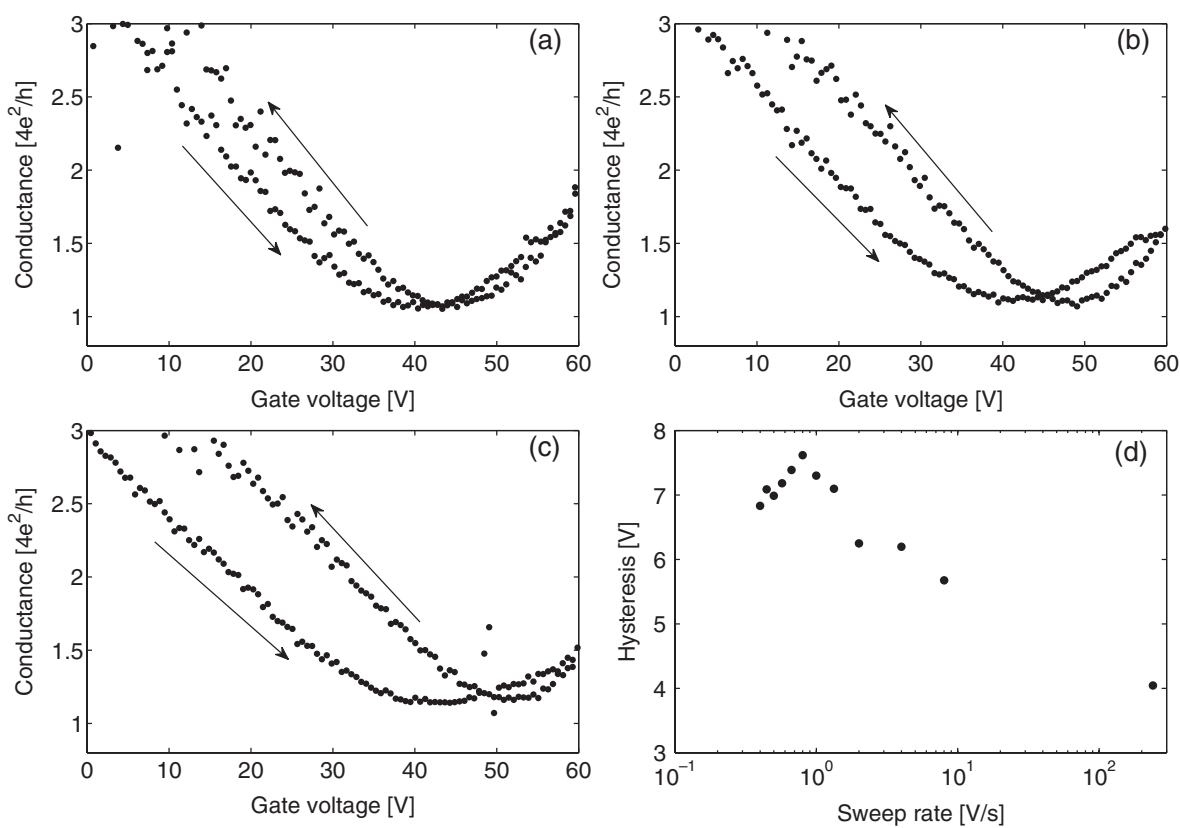

Figure 3. Measurements in a vacuum showing conductance (in $4 e^{2} / h$ ) as a function of applied gate voltage for different sweep rates: (a) $240 \mathrm{~V} \mathrm{~s}^{-1}$, (b) $4 \mathrm{~V} \mathrm{~s}^{-1}$, (c) $0.4 \mathrm{~V} \mathrm{~s}^{-1}$. Arrows indicate the sweep direction. The hysteresis appears to increase as the sweep rate is decreased. (d) Hysteresis as function of sweep rate, which shows a maximum in hysteresis at $0.8 \mathrm{~V} \mathrm{~s}-1$. Hysteresis was found by a parabolic fit to the data below a conductance of 1.5 times $4 e^{2} / h$. Error on the fit is negligible. 

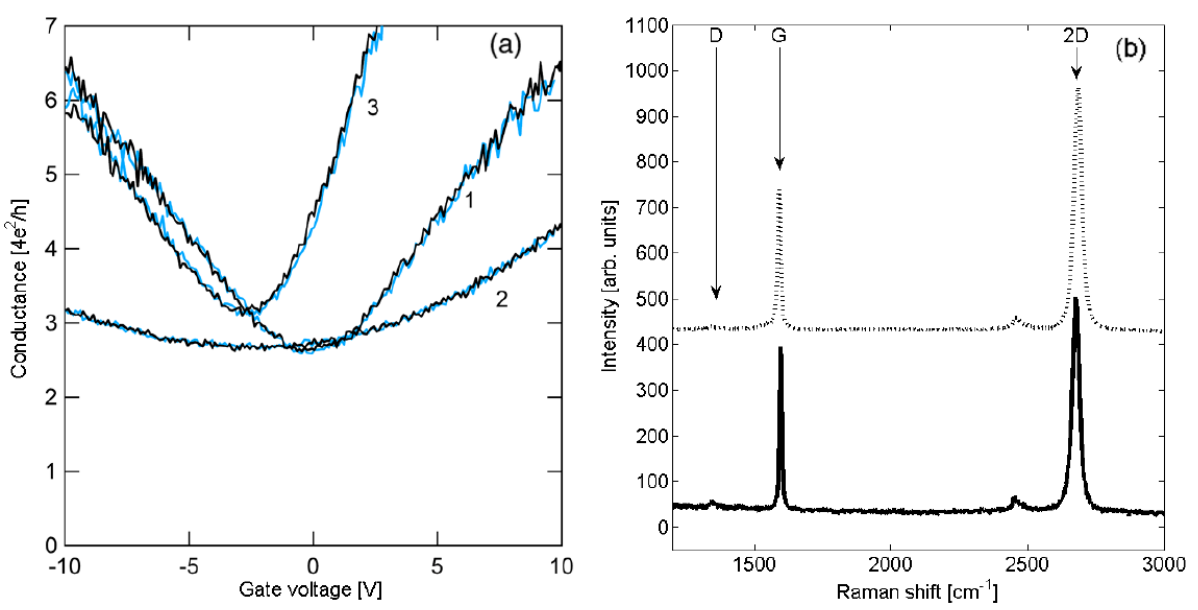

Figure 4. (a) Environmental measurement where the mobility decreases when graphene was exposed to water vapor. Graphene starts in high vacuum (1), water vapor is introduced (low vacuum) (2) and finally high vacuum is achieved again (3). Colors represent up- (black) and down-sweep (blue). (b) Raman spectrum before (solid) and after (dotted) measurements in SEM. The spectrum is shifted for clarity. The ratio of the intensity of the D peak to the G peak remains small after the measurement, indicating that the graphene did not alter in structure using the method described in the text.

The charge neutrality points are calculated by fitting a parabola near the minimum conductance of the up- and downsweep, and finding the minimum of the fit. The carrier mobility is estimated in the same manner as the measurements in ambient conditions. As the sweep rate is decreased, the hysteresis changes, and the mobilities are observed to decrease slightly from $\sim 1200$ to $\sim 1000 \mathrm{~cm}^{2} \mathrm{~V}^{-1} \mathrm{~s}^{-1}$.

The variation of hysteresis with sweep time is shown in figure 3(d). It is observed that the hysteresis increases up to $0.8 \mathrm{~V} \mathrm{~s}^{-1}$, followed by a decrease. This trend was observed on all samples investigated.

In the following work we use an environmental SEM in which a range of gases can be leaked into the sample chamber in order to study the influence of the local gas environment on conductance and mobility. Figure 4(a) shows the effect of water vapor on conductance. First, a high vacuum $\left(10^{-5} \mathrm{~Pa}\right)$ measurement was performed (1), resulting in mobility around $2000 \mathrm{~cm}^{2} \mathrm{~V}^{-1} \mathrm{~s}^{-1}$. Water vapor was introduced at $400 \mathrm{~Pa}$ (2), and the mobility dropped to roughly $500 \mathrm{~cm}^{2} \mathrm{~V}^{-1} \mathrm{~s}^{-1}$. Evacuating the chamber to $10^{-5} \mathrm{~Pa}$ again (3) the mobility was restored to its initial value, with a slight shift in the charge neutrality point and minimum conductivity.

Figure 4(b) shows Raman spectra from pristine graphene samples and graphene samples contacted outside the field of view. The $I(\mathrm{D}) / I(\mathrm{G})$ ratio remains small after ESEM measurements, which shows no indication of an increased density of defects [35].

Our results obtained under vacuum in a SEM show that the hysteresis in the charge neutrality point depends on the sweep rate, with a maximum hysteresis at $0.8 \mathrm{~V} \mathrm{~s}^{-1}$. The hysteresis effect has been observed in many graphene field effect devices, and has been suggested to be caused by charge trapping in the $\mathrm{SiO}_{2}[4,30]$.

When changing the gate voltage, the electric field traps or detraps charges at a specific rate. Therefore, it may be expected that the hysteresis will approach zero for high sweep rates since the traps do not have time to fill. This should lead to a timedependence of the hysteretic effect, and thus a varying offset of the charge neutrality point. Furthermore, it is expected that a steady state will be achieved for very low sweep rates, so hysteresis should also approach a constant value, possibly zero. Mattmann et al [36] demonstrated that pulsing the gate voltage is a highly efficient method to strongly reduce gate hysteresis for carbon nanotube based field effect sensors.

Environmental measurements showed a clear reduction in carrier mobility, when comparing high vacuum to low vacuum with water vapor. These results contradict previous measurements with a variety of adsorbed gases on graphene which showed no change in mobility [37]. We are unable to specifically determine the cause of this contradiction, but suggest that our observations of changing mobility may be due to ionization of water molecules which adsorb onto the graphene surface, and thereby increase the number of charged surface scatterers. Protons and hydroxyl groups may also be able to neutralize some of the charged species already on the surface, which then may desorb from the surface when a high vacuum is reintroduced. This would explain why the mobility is slightly increased when comparing the two high vacuum states.

While we frequently encountered a poor electrical contact to the graphene, damage to the graphene surface was rarely observed. The graphene seemed very resistant to mechanical scratching; however, breakdown occurred consistently at a current of around $100 \mu \mathrm{A}$ or more. This can be explained by a high current density at the contact points, which from previous studies [38] can be expected to be at minimum $100 \mathrm{~nm}$ in diameter. Assuming the thickness of the graphene layer to be 3.4 $\AA$, the current density at the perimeter of a contact area is in rough agreement with the critical current density of graphene of $10^{8} \mathrm{~A} \mathrm{~cm}^{-2}$ found experimentally by Murali et al [39], at $10^{7} \mathrm{~A} \mathrm{~cm}^{-2}$. 


\section{Conclusion}

We have shown that micro four-point probes can be used to perform local and non-destructive measurements on graphene, within a shorter time span than with commonly used lithographic processing. The sample size is only limited by the electrode pitch, and the non-destructive approach makes further processing of the sample possible. To compete with lithographic contacts, it is of key importance that the approach can be used in a vacuum or controlled atmospheric conditions, which we have demonstrated here. We observed a strong tendency of the carrier mobility to decrease in the presence of water vapor, which conflicts with previous reports. It is anticipated that micro four-point probes could be an invaluable tool for inline verification and process monitoring in graphene fabrication, as well as providing a unique possibility of probing and mapping the local transport properties of graphene.

\section{Acknowledgments}

We appreciate financial support by the Danish Research Council for Technology and Production Sciences (Nanoengineered Graphene Devices) as well as helpful discussions with Cosmin Roman and Christofer Hierold, both ETH Zurich, Micro and Nanosystems.

\section{References}

[1] Novoselov K S, Geim A K, Morozov S V, Jiang D, Zhang Y, Dubonos S V, Grigorieva I V and Firsov A A 2004 Electric field effect in atomically thin carbon films Science 306 666-9

[2] Avouris P, Chen Z and Perebeinos V 2007 Carbon-based electronics Nature Nanotechnol. 2 605-15

[3] Novoselov K S, Geim A K, Morozov S V, Jiang D, Katsnelson M I, Grigorieva I V, Dubonos S V and Firsov A A 2005 Two-dimensional gas of massless Dirac fermions in graphene Nature 438 197-200

[4] Lafkioti M, Krauss B, Lohmann T, Zschieschang U, Klauk H, Klitzing K and Smet J H 2010 Graphene on a hydrophobic substrate: doping reduction and hysteresis suppression under ambient conditions Nano Lett. 10 1149-53

[5] Lohmann T, Klitzing K and Smet J H 2009 Four-terminal magneto-transport in graphene $\mathrm{p}-\mathrm{n}$ junctions created by spatially selective doping Nano Lett. 9 1973-9

[6] Cao $\mathrm{H}$ et al 2010 Electronic transport in chemical vapor deposited graphene synthesized on $\mathrm{Cu}$ : quantum Hall effect and weak localization Appl. Phys. Lett. 96122106

[7] Bae S et al 2010 Roll-to-roll production of 30-inch graphene films for transparent electrodes Nature Nanotechnol. 5 574-8

[8] Li X et al 2009 Large-area synthesis of high-quality and uniform graphene films on copper foils Science 324 1312-4

[9] Ishigami M, Chen J H, Cullen W G, Fuhrer M S and Williams E D 2007 Atomic structure of graphene on $\mathrm{SiO}_{2}$ Nano Lett. 7 1643-8

[10] Jacobsen A, Koehler F M, Stark W J and Ensslin K 2010 Towards electron transport measurements in chemically modified graphene: effect of a solvent New J. Phys. 12125007

[11] Yu Y, Zhao Y, Ryu S, Brus L E, Kim K S and Kim P 2009 Tuning the graphene work function by electric field effect Nano Lett. $93430-4$
[12] Filleter T, Emtsev K V, Seyller Th and Bennewitz R 2008 Local work function measurements of epitaxial graphene Appl. Phys. Lett. 93133117

[13] Kellar J A, Alaboson J M P, Wang Q H and Hersam M C 2010 Identifying and characterizing epitaxial graphene domains on partially graphitized $\mathrm{SiC}(0001)$ surfaces using scanning probe microscopy Appl. Phys. Lett. 96143103

[14] Yakes M K, Gunlycke D, Tedesco J L, Campbell P M, Myers-Ward R L, Eddy C R, Gaskill D K, Sheehan P E and Laracuente A R 2010 Conductance anisotropy in epitaxial graphene sheets generated by substrate interactions Nano Lett. 10 1559-62

[15] Kanagawa T, Hobara R, Matsuda I, Tanikawa T, Natori A and Hasegawa S 2003 Anisotropy in conductance of a quasi-one-dimensional metallic surface state measured by a square micro four-point probe method Phys. Rev. Lett. 91036805

[16] Petersen C L, Grey F, Shiraki I and Hasegawa S 2000 Microfour-point probe for studying electronic transport through surface states Appl. Phys. Lett. 773782

[17] Bøggild P, Grey F, Hassenkam T, Greve D R and Bjørnholm T 2000 Direct measurement of the microscale conductivity of conjugated polymer monolayers Adv. Mater. 12 947-50

[18] Rosseel E et al 2009 Monitoring of local and global temperature non-uniformities by means of therma-probe and micro four-point probe metrology 17th Int. Conf. on Advanced Thermal Processing of Semiconductors pp 1-6

[19] Petersen D H, Hansen O, Lin R and Nielsen P F 2008 Micro-four-point probe Hall effect measurement method J. Appl. Phys. 104013710

[20] Bolotin K I, Sikes K J, Jiang Z, Klima M, Fudenberg G, Hone J, Kim P and Stormer H L 2008 Ultrahigh electron mobility in suspended graphene Solid State Commun. 146 351-5

[21] Huard B, Stander J, Sulpizio J A and Goldhaber-Gordon D 2008 Evidence of the role of contacts on the observed electron-hole asymmetry in graphene Phys. Rev. B 78 121405(R)

[22] Venugopal A, Colombo L and Vogel E M 2010 Contact resistance in few and multilayer graphene devices Appl. Phys. Lett. 96013512

[23] Alwarappan S, Erdem A, Liu C and Li C 2009 Probing the electrochemical properties of graphene nanosheets for biosensing applications J. Phys. Chem. C 20 8853-6

[24] Smits F M 1958 Measurement of sheet resistivities with the four-point probe Bell Syst. Techn. J. 37 711-8

[25] Rymaszewski R 1969 Relationship between the correction factor of the four-point probe value and the selection of potential and current electrode J. Phys. E: Sci. Instrum. 2170

[26] Thorsteinsson S, Wang F, Petersen D H, Hansen T M, Kjaer D, Lin R, Kim J Y, Nielsen P F and Hansen O 2009 Accurate microfour-point probe sheet resistance measurements on small samples Rev. Sci. Instrum. 80053902

[27] Dan Y, Lu Y, Kybert N J, Luo Z and Johnson A T C 2009 Intrinsic response of graphene vapor sensors Nano Lett. 9 1472-5

[28] Joshi P, Romero H E, Neal A T, Toutam V K and Tadigadapa S A 2010 Intrinsic doping and gate hysteresis in graphene field effect devices fabricated on $\mathrm{SiO}_{2}$ substrates J. Phys.: Condens. Matter 22334214

[29] Liao Z, Han B, Zhou Y and Yu D 2010 Hysteresis reversion in graphene field-effect transistors J. Chem. Phys. 133044703

[30] Wang H, Wu Y, Cong C, Shang J and Yu T 2010 Hysteresis of electronic transport in graphene transistors ACS Nano $47221-8$ 
[31] Teweldebrhan D and Balandin A A 2009 Modification of graphene properties due to electron-beam irradiation Appl. Phys. Lett. 94013101

[32] Rao G, Mctaggart S, Lee J L and Geer R E 2009 Study of electron beam irradiation induced defectivity in mono and bi layer graphene and the influence on raman band position and line-width Mater. Res. Soc. Symp. Proc. 1184 151-6

[33] Childres I, Jauregui L A, Foxe M, Tian J, Jalilian R, Jovanovic I and Chen Y P 2010 Effect of electron-beam irradiation on graphene field effect devices Appl. Phys. Lett. 97173109

[34] Xu M, Fujita D and Hanagata N 2010 Monitoring electron-beam irradiation effects on graphene by temporal Auger electron spectroscopy Nanotechnology 21265705

[35] Tang B, Hu G and Hanyang G 2010 Raman spectroscopic characterization of graphene Appl. Spectrosc. Rev. 45 369-407
[36] Mattmann M, Roman C, Helbling T, Bechstein D, Durrer L, Pohle R, Fleischer M and Hierold C 2010 Pulsed gate sweep strategies for hysteresis reduction in carbon nanotube transistors for low concentration $\mathrm{NO}_{2}$ gas detection Nanotechnology 21185501

[37] Schedin F, Geim A K, Morozov S V, Hill E W, Blake P, Katsnelson M I and Novoselov K S 2007 Detection of individual gas molecules adsorbed on graphene Nature Mater. 6 652-5

[38] Ansbaek T, Petersen D H, Hansen O, Larsen J B, Hansen T M and Bøggild P 2009 Fundamental size limitations of micro four-point probes Microelectron. Eng. 86 987-90

[39] Murali R, Yang Y, Brenner K, Beck T and Meindl J D 2009 Breakdown current density of graphene nanoribbons Appl. Phys. Lett. 94243114 